Network Working Group

Request for Comments: 5077

Obsoletes: 4507

Category: Standards Track
J. Salowey

H. Zhou

Cisco Systems

P. Eronen

Nokia

H. Tschofenig

Nokia Siemens Networks

January 2008

\title{
Transport Layer Security (TLS) Session Resumption without Server-Side State
}

\section{Status of This Memo}

This document specifies an Internet standards track protocol for the Internet community, and requests discussion and suggestions for improvements. Please refer to the current edition of the "Internet Official Protocol Standards" (STD 1) for the standardization state and status of this protocol. Distribution of this memo is unlimited.

Abstract

This document describes a mechanism that enables the Transport Layer Security (TLS) server to resume sessions and avoid keeping per-client session state. The TLS server encapsulates the session state into a ticket and forwards it to the client. The client can subsequently resume a session using the obtained ticket. This document obsoletes RFC 4507. 
Table of Contents

1. Introduction . . . . . . . . . . . . . . . . . . . . . . . 3

2. Terminology . . . . . . . . . . . . . . . . . . . . . . . . . 3

3. Protocol . . . . . . . . . . . . . . . . . . . . . . . 3

3.1. Overview . . . . . . . . . . . . . . . . . . . . . . . 4 4

3.2. SessionTicket TLS Extension . . . . . . . . . . . . . . 7

3.3. NewSessionTicket Handshake Message . . . . . . . . . . . . 8

3.4. Interaction with TLS Session ID . . . . . . . . . . . . . 9

4. Recommended Ticket Construction . . . . . . . . . . . . . . 10

5. Security Considerations . . . . . . . . . . . . . . . . . . 12

5.1. Invalidating Sessions . . . . . . . . . . . . . . . . 12

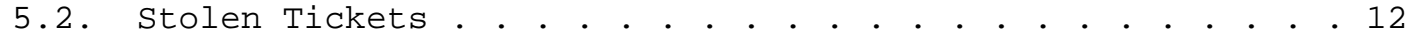

5.3. Forged Tickets . . . . . . . . . . . . . . . . . . . . 12

5.4. Denial of Service Attacks . . . . . . . . . . . . . . . . 12

5.5. Ticket Protection Key Management . . . . . . . . . . . . . 13

5.6. Ticket Lifetime . . . . . . . . . . . . . . . . . . . . . 13

5.7. Alternate Ticket Formats and Distribution Schemes . . . 13

5.8. Identity Privacy, Anonymity, and Unlinkability . . . . . . 14

6. Acknowledgements . . . . . . . . . . . . . . . . . . 14

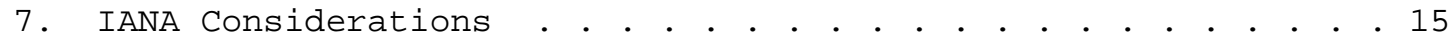

8. References . . . . . . . . . . . . . . . . . . . . . . 15

8.1. Normative References . . . . . . . . . . . . . . . 15

8.2. Informative References . . . . . . . . . . . . . . . . 15

Appendix A. Discussion of Changes to RFC 4507 . . . . . . . . . 17 


\section{Introduction}

This document defines a way to resume a Transport Layer security (TLS) session without requiring session-specific state at the TLS server. This mechanism may be used with any TLS ciphersuite. This document applies to both TLS 1.0 defined in [RFC2246], and TLS 1.1 defined in [RFC4346]. The mechanism makes use of TLS extensions defined in [RFC4366] and defines a new TLS message type.

This mechanism is useful in the following situations:

1. servers that handle a large number of transactions from different users

2. servers that desire to cache sessions for a long time

3. ability to load balance requests across servers

4. embedded servers with little memory

This document obsoletes RFC 4507 [RFC4507] to correct an error in the encoding that caused the specification to differ from deployed implementations. At the time of this writing, there are no known implementations that follow the encoding specified in RFC 4507. This update to RFC 4507 aligns the document with currently deployed implementations. More details of the change are given in Appendix A.

2. Terminology

Within this document, the term 'ticket' refers to a cryptographically protected data structure that is created and consumed by the server to rebuild session-specific state.

The key words "MUST", "MUST NOT", "REQUIRED", "SHALL", "SHALL NOT", "SHOULD", "SHOULD NOT", "RECOMMENDED", "MAY", and "OPTIONAL" in this document are to be interpreted as described in [RFC2119].

\section{Protocol}

This specification describes a mechanism to distribute encrypted session-state information to the client in the form of a ticket and a mechanism to present the ticket back to the server. The ticket is created by a TLS server and sent to a TLS client. The TLS client presents the ticket to the TLS server to resume a session. Implementations of this specification are expected to support both mechanisms. Other specifications can take advantage of the session tickets, perhaps specifying alternative means for distribution or selection. For example, a separate specification may describe an 
alternate way to distribute a ticket and use the TLS extension in this document to resume the session. This behavior is beyond the scope of the document and would need to be described in a separate specification.

\subsection{Overview}

The client indicates that it supports this mechanism by including a SessionTicket TLS extension in the ClientHello message. The extension will be empty if the client does not already possess a ticket for the server. The server sends an empty sessionticket extension to indicate that it will send a new session ticket using the NewSessionTicket handshake message. The extension is described in Section 3.2.

If the server wants to use this mechanism, it stores its session state (such as ciphersuite and master secret) to a ticket that is encrypted and integrity-protected by a key known only to the server. The ticket is distributed to the client using the NewSessionTicket TLS handshake message described in section 3.3. This message is sent during the TLS handshake before the ChangeCipherSpec message, after the server has successfully verified the client's Finished message.

Client

Server

ClientHello (empty SessionTicket extension)-------->

ServerHello (empty Sessionticket extension) Certificate* ServerkeyExchange* CertificateRequest*

Certificate* <-------- ServerHelloDone

ClientKeyExchange CertificateVerify* [ChangeCipherSpec] Finished

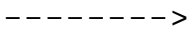

NewSessionTicket [ChangeCipherSpec] Finished Application Data

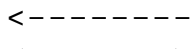
$<------->$ Application Data

Figure 1: Message Flow for Full Handshake Issuing New Session Ticket 
The client caches this ticket along with the master secret and other parameters associated with the current session. When the client wishes to resume the session, it includes the ticket in the Sessionticket extension within the ClientHello message. Appendix A provides a detailed description of the encoding of the extension and changes from RFC 4507. The server then decrypts the received ticket, verifies the ticket's validity, retrieves the session state from the contents of the ticket, and uses this state to resume the session. The interaction with the TLS session ID is described in section 3.4. If the server successfully verifies the client's ticket, then it may renew the ticket by including a NewSessionTicket handshake message after the serverHello.

Client

Server

ClientHello

(SessionTicket extension)

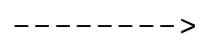

ServerHello

(empty SessionTicket extension)

NewSessionTicket [ChangeCipherSpec]

[ChangeCipherSpec]

Finished

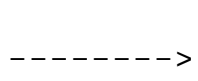

Application Data

$<------>\quad$ Application Data

Figure 2: Message Flow for Abbreviated Handshake Using New Session Ticket

A recommended ticket format is given in section 4.

If the server cannot or does not want to honor the ticket, then it can initiate a full handshake with the client.

In the case that the server does not wish to issue a new ticket at this time, it just completes the handshake without including a Sessionticket extension or NewSessionTicket handshake message. This is shown below (this flow is identical to Figure 1 in RFC 4346, except for the sessionTicket extension in the first message): 


\title{
Client
}

Server

ClientHello

(SessionTicket extension)

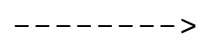

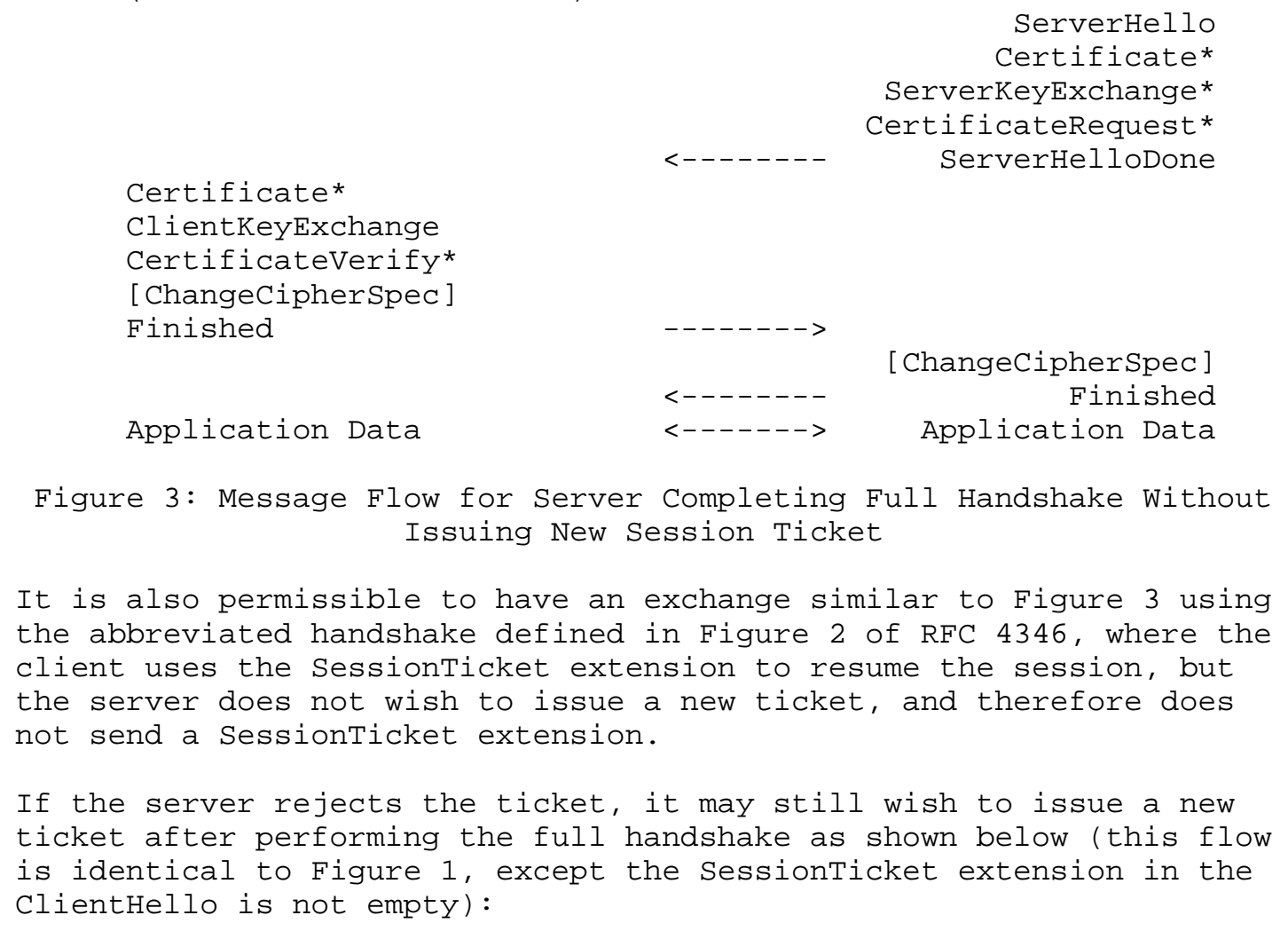

ServerHello ServerKeyExchange* ificateRequest*

Certificate* ClientKeyExchange CertificateVerify * [ChangeCipherSpec]

Finished Finished Application Data

Figure 3: Message Flow for Server Completing Full Handshake Without Issuing New Session Ticket

\begin{abstract}
It is also permissible to have an exchange similar to Figure 3 using the abbreviated handshake defined in Figure 2 of RFC 4346, where the client uses the SessionTicket extension to resume the session, but the server does not wish to issue a new ticket, and therefore does ticket after performing the full handshake as shown below (this flow Clienthello is not empty):
\end{abstract}




\section{Client}

Server

ClientHello

(SessionTicket extension)

$-------->$

ServerHello

(empty Sessionticket extension)

Certificate* ServerkeyExchange* CertificateRequest*

Certificate* <------- ServerHelloDone

ClientKeyExchange

CertificateVerify * [ChangeCipherSpec] Finished

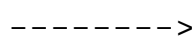

Application Data

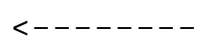

$<------>$
NewSessionTicket [ChangeCipherSpec]

Finished

Application Data

Figure 4: Message Flow for Server Rejecting Ticket, Performing Full Handshake, and Issuing New Session Ticket

\subsection{Sessionticket TLS Extension}

The SessionTicket TLS extension is based on [RFC4366]. The format of the ticket is an opaque structure used to carry session-specific state information. This extension may be sent in the clientHello and ServerHello.

If the client possesses a ticket that it wants to use to resume a session, then it includes the ticket in the SessionTicket extension in the ClientHello. If the client does not have a ticket and is prepared to receive one in the NewSessionTicket handshake message, then it MUST include a zero-length ticket in the SessionTicket extension. If the client is not prepared to receive a ticket in the NewSessionTicket handshake message, then it MUST NOT include a Sessionticket extension unless it is sending a non-empty ticket it received through some other means from the server.

The server uses a zero-length SessionTicket extension to indicate to the client that it will send a new session ticket using the NewSessionTicket handshake message described in section 3.3. The server MUST send this extension in the ServerHello if it wishes to issue a new ticket to the client using the NewSessionTicket handshake message. The server MUST NOT send this extension if it does not receive one in the clientHello. 
If the server fails to verify the ticket, then it falls back to performing a full handshake. If the ticket is accepted by the server but the handshake fails, the client sHOULD delete the ticket.

The Sessionticket extension has been assigned the number 35 . The extension_data field of Sessionticket extension contains the ticket.

\subsection{NewSessionTicket Handshake Message}

This message is sent by the server during the TLS handshake before the ChangeCipherSpec message. This message MUST be sent if the server included a SessionTicket extension in the ServerHello. This message MUST NOT be sent if the server did not include a SessionTicket extension in the ServerHello. This message is included in the hash used to create and verify the Finished message. In the case of a full handshake, the server MUST verify the client's Finished message before sending the ticket. The client MUST NOT treat the ticket as valid until it has verified the server's Finished message. If the server determines that it does not want to include a ticket after it has included the sessionTicket extension in the ServerHello, then it sends a zero-length ticket in the NewSessionticket handshake message.

If the server successfully verifies the client's ticket, then it MAY renew the ticket by including a NewSessionTicket handshake message after the ServerHello in the abbreviated handshake. The client should start using the new ticket as soon as possible after it verifies the server's Finished message for new connections. Note that since the updated ticket is issued before the handshake completes, it is possible that the client may not put the new ticket into use before it initiates new connections. The server MUST NOT assume that the client actually received the updated ticket until it successfully verifies the client's Finished message.

The NewSessionTicket handshake message has been assigned the number 4 and its definition is given at the end of this section. The ticket_lifetime_hint field contains a hint from the server about how long the ticket should be stored. The value indicates the lifetime in seconds as a 32-bit unsigned integer in network byte order relative to when the ticket is received. A value of zero is reserved to indicate that the lifetime of the ticket is unspecified. A client SHOULD delete the ticket and associated state when the time expires. It MAY delete the ticket earlier based on local policy. A server MAY treat a ticket as valid for a shorter or longer period of time than what is stated in the ticket_lifetime_hint. 


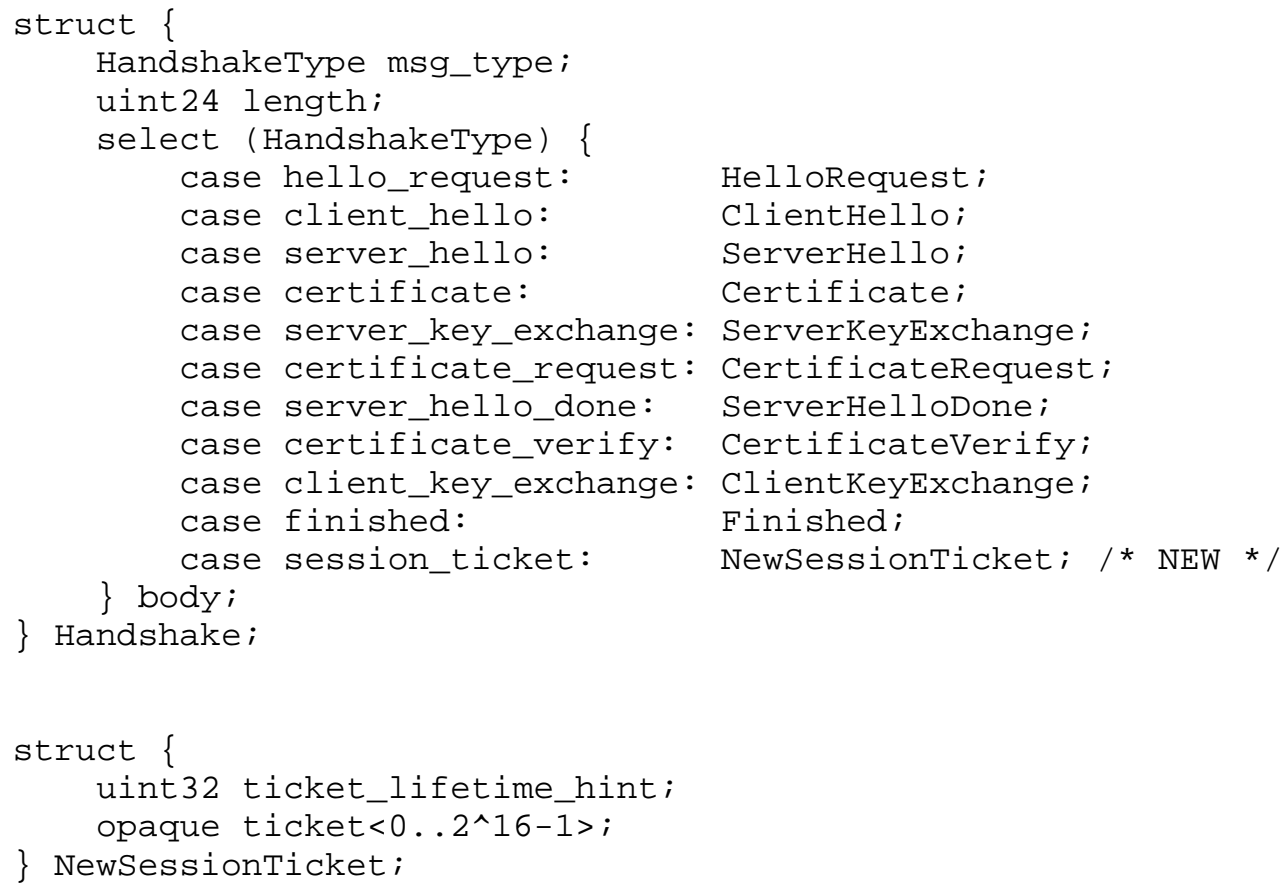

\subsection{Interaction with TLS Session ID}

If a server is planning on issuing a session ticket to a client that does not present one, it SHOULD include an empty session ID in the ServerHello. If the server rejects the ticket and falls back to the full handshake then it may include a non-empty session ID to indicate its support for stateful session resumption. If the client receives a session ticket from the server, then it discards any session ID that was sent in the serverHello.

When presenting a ticket, the client MAY generate and include a Session ID in the TLS ClientHello. If the server accepts the ticket and the session ID is not empty, then it MUST respond with the same Session ID present in the ClientHello. This allows the client to easily differentiate when the server is resuming a session from when it is falling back to a full handshake. Since the client generates a Session ID, the server MUST NOT rely upon the session ID having a particular value when validating the ticket. If a ticket is presented by the client, the server MUST NOT attempt to use the Session ID in the ClientHello for stateful session resumption. Alternatively, the client MAY include an empty session ID in the clientHello. In this case, the client ignores the session ID sent in the ServerHello and determines if the server is resuming a session by the subsequent handshake messages. 
4. Recommended Ticket Construction

This section describes a recommended format and protection for the ticket. Note that the ticket is opaque to the client, so the structure is not subject to interoperability concerns, and implementations may diverge from this format. If implementations do diverge from this format, they must take security concerns seriously. Clients MUST NOT examine the ticket under the assumption that it complies with this document.

The server uses two different keys: one 128-bit key for Advanced Encryption Standard (AES) [AES] in Cipher Block Chaining (CBC) mode [CBC] encryption and one 256-bit key for HMAC-SHA-256 [RFC4634].

The ticket is structured as follows:

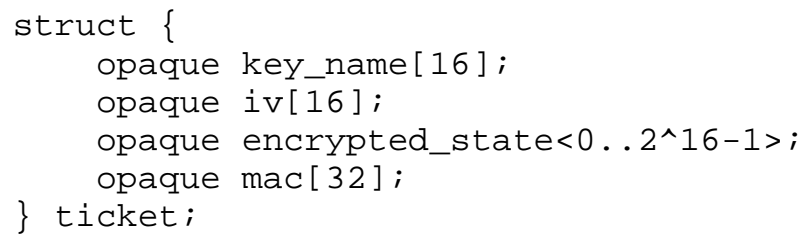

Here, key_name serves to identify a particular set of keys used to protect the ticket. It enables the server to easily recognize tickets it has issued. The key_name should be randomly generated to avoid collisions between servers. One possibility is to generate new random keys and key_name every time the server is started.

The actual state information in encrypted_state is encrypted using 128-bit AES in CBC mode with the given IV. The Message Authentication Code (MAC) is calculated using HMAC-SHA-256 over key_name (16 octets) and IV (16 octets), followed by the length of the encrypted_state field (2 octets) and its contents (variable length). 


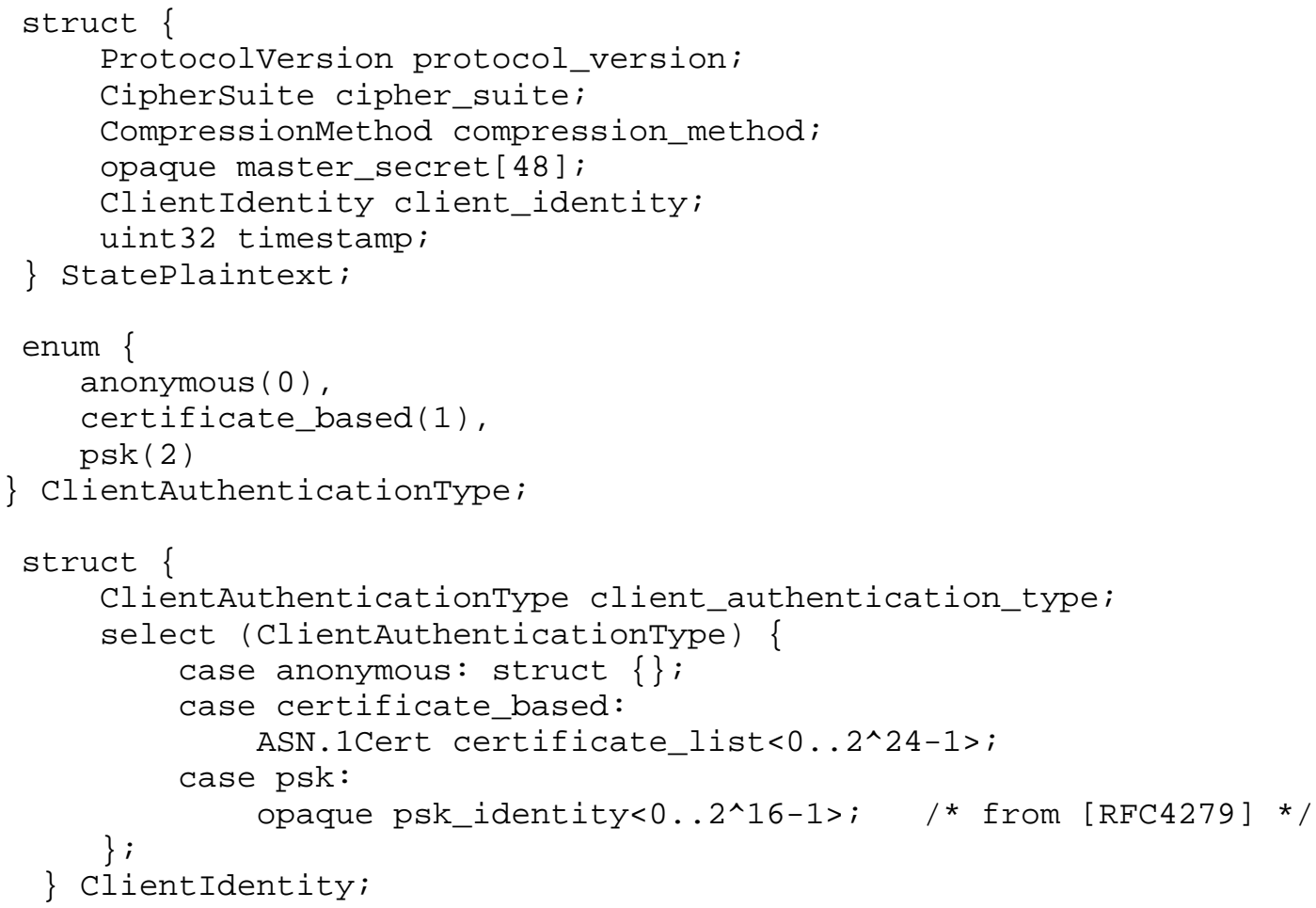




\section{Security Considerations}

This section addresses security issues related to the usage of a ticket. Tickets must be authenticated and encrypted to prevent modification or eavesdropping by an attacker. Several attacks described below will be possible if this is not carefully done.

Implementations should take care to ensure that the processing of tickets does not increase the chance of denial of service as described below.

\subsection{Invalidating Sessions}

The TLS specification requires that TLS sessions be invalidated when errors occur. [CSSC] discusses the security implications of this in detail. In the analysis within this paper, failure to invalidate sessions does not pose a security risk. This is because the TLS handshake uses a non-reversible function to derive keys for a session so information about one session does not provide an advantage to attack the master secret or a different session. If a session invalidation scheme is used, the implementation should verify the integrity of the ticket before using the contents to invalidate a session to ensure that an attacker cannot invalidate a chosen session.

\subsection{Stolen Tickets}

An eavesdropper or man-in-the-middle may obtain the ticket and attempt to use it to establish a session with the server; however, since the ticket is encrypted and the attacker does not know the secret key, a stolen ticket does not help an attacker resume a session. A TLS server MUST use strong encryption and integrity protection for the ticket to prevent an attacker from using a brute force mechanism to obtain the ticket's contents.

\subsection{Forged Tickets}

A malicious user could forge or alter a ticket in order to resume a session, to extend its lifetime, to impersonate another user, or to gain additional privileges. This attack is not possible if the ticket is protected using a strong integrity protection algorithm such as a keyed HMAC-SHA-256.

\subsection{Denial of Service Attacks}

The key_name field defined in the recommended ticket format helps the server efficiently reject tickets that it did not issue. However, an adversary could store or generate a large number of tickets to send 
to the TLS server for verification. To minimize the possibility of a denial of service, the verification of the ticket should be lightweight (e.g., using efficient symmetric key cryptographic algorithms).

\subsection{Ticket Protection Key Management}

A full description of the management of the keys used to protect the ticket is beyond the scope of this document. A list of RECOMMENDED practices is given below.

- The keys should be generated securely following the randomness recommendations in [RFC4086].

- The keys and cryptographic protection algorithms should be at least 128 bits in strength. Some ciphersuites and applications may require cryptographic protection greater than 128 bits in strength.

- The keys should not be used for any purpose other than generating and verifying tickets.

o The keys should be changed regularly.

- The keys should be changed if the ticket format or cryptographic protection algorithms change.

\subsection{Ticket Lifetime}

The TLS server controls the lifetime of the ticket. Servers determine the acceptable lifetime based on the operational and security requirements of the environments in which they are deployed. The ticket lifetime may be longer than the 24-hour lifetime recommended in [RFC4346]. TLS clients may be given a hint of the lifetime of the ticket. Since the lifetime of a ticket may be unspecified, a client has its own local policy that determines when it discards tickets.

\subsection{Alternate Ticket Formats and Distribution Schemes}

If the ticket format or distribution scheme defined in this document is not used, then great care must be taken in analyzing the security of the solution. In particular, if confidential information, such as a secret key, is transferred to the client, it MUST be done using secure communication so as to prevent attackers from obtaining or modifying the key. Also, the ticket MUST have its integrity and confidentiality protected with strong cryptographic techniques to prevent a breach in the security of the system. 


\subsection{Identity Privacy, Anonymity, and Unlinkability}

This document mandates that the content of the ticket is confidentiality protected in order to avoid leakage of its content, such as user-relevant information. As such, it prevents disclosure of potentially sensitive information carried within the ticket.

The initial handshake exchange, which was used to obtain the ticket, might not provide identity confidentiality of the client based on the properties of TLS. Another relevant security threat is the ability for an on-path adversary to observe multiple TLS handshakes where the same ticket is used, therefore concluding they belong to the same communication endpoints. Application designers that use the ticket mechanism described in this document should consider that unlinkability [ANON] is not necessarily provided.

While a full discussion of these topics is beyond the scope of this document, it should be noted that it is possible to issue a ticket using a TLS renegotiation handshake that occurs after a secure tunnel has been established by a previous handshake. This may help address some privacy and unlinkability issues in some environments.

6. Acknowledgements

The authors would like to thank the following people for their help with preparing and reviewing this document: Eric Rescorla, Mohamad Badra, Tim Dierks, Nelson Bolyard, Nancy Cam-Winget, David McGrew, Rob Dugal, Russ Housley, Amir Herzberg, Bernard Aboba, and members of the TLS working group.

[CSSC] describes a solution that is very similar to the one described in this document and gives a detailed analysis of the security considerations involved. [RFC2712] describes a mechanism for using Kerberos [RFC4120] in TLS ciphersuites, which helped inspire the use of tickets to avoid server state. [RFC4851] makes use of a similar mechanism to avoid maintaining server state for the cryptographic tunnel. [SC97] also investigates the concept of stateless sessions.

The authors would also like to thank Jan Nordqvist, who found the encoding error in RFC 4507, corrected by this document. In addition Nagendra Modadugu, Wan-Teh Chang, and Michael D'Errico provided useful feedback during the review of this document. 
7. IANA Considerations

IANA has assigned a TLS extension number of 35 to the SessionTicket TLS extension from the TLS registry of ExtensionType values defined in $[\mathrm{RFC} 4366]$.

IANA has assigned a TLS HandshakeType number 4 to the NewSessionTicket handshake type from the TLS registry of HandshakeType values defined in [RFC4346].

This document does not require any actions or assignments from IANA.

8. References

8.1. Normative References

[RFC2119] Bradner, S., "Key words for use in RFCs to Indicate Requirement Levels", BCP 14, RFC 2119, March 1997.

[RFC2246] Dierks, T. and C. Allen, "The TLS Protocol Version 1.0", RFC 2246, January 1999.

[RFC4346] Dierks, T. and E. Rescorla, "The Transport Layer Security (TLS) Protocol Version 1.1", RFC 4346, April 2006.

[RFC4366] Blake-Wilson, S., Nystrom, M., Hopwood, D., Mikkelsen, J., and T. Wright, "Transport Layer Security (TLS) Extensions", RFC 4366, April 2006.

[RFC4507] Salowey, J., Zhou, H., Eronen, P., and H. Tschofenig, "Transport Layer Security (TLS) Session Resumption without Server-Side State", RFC 4507, May 2006.

8.2. Informative References

[AES] National Institute of Standards and Technology, "Advanced Encryption Standard (AES)", Federal Information Processing Standards (FIPS) Publication 197, November 2001.

[ANON] Pfitzmann, A. and M. Hansen, "Anonymity, Unlinkability, Unobservability, Pseudonymity, and Identity Management - A Consolidated Proposal for Terminology", http:// dud.inf.tu-dresden.de/literatur/ Anon_Terminology_v0.26-1.pdf Version 0.26, December 2005 . 


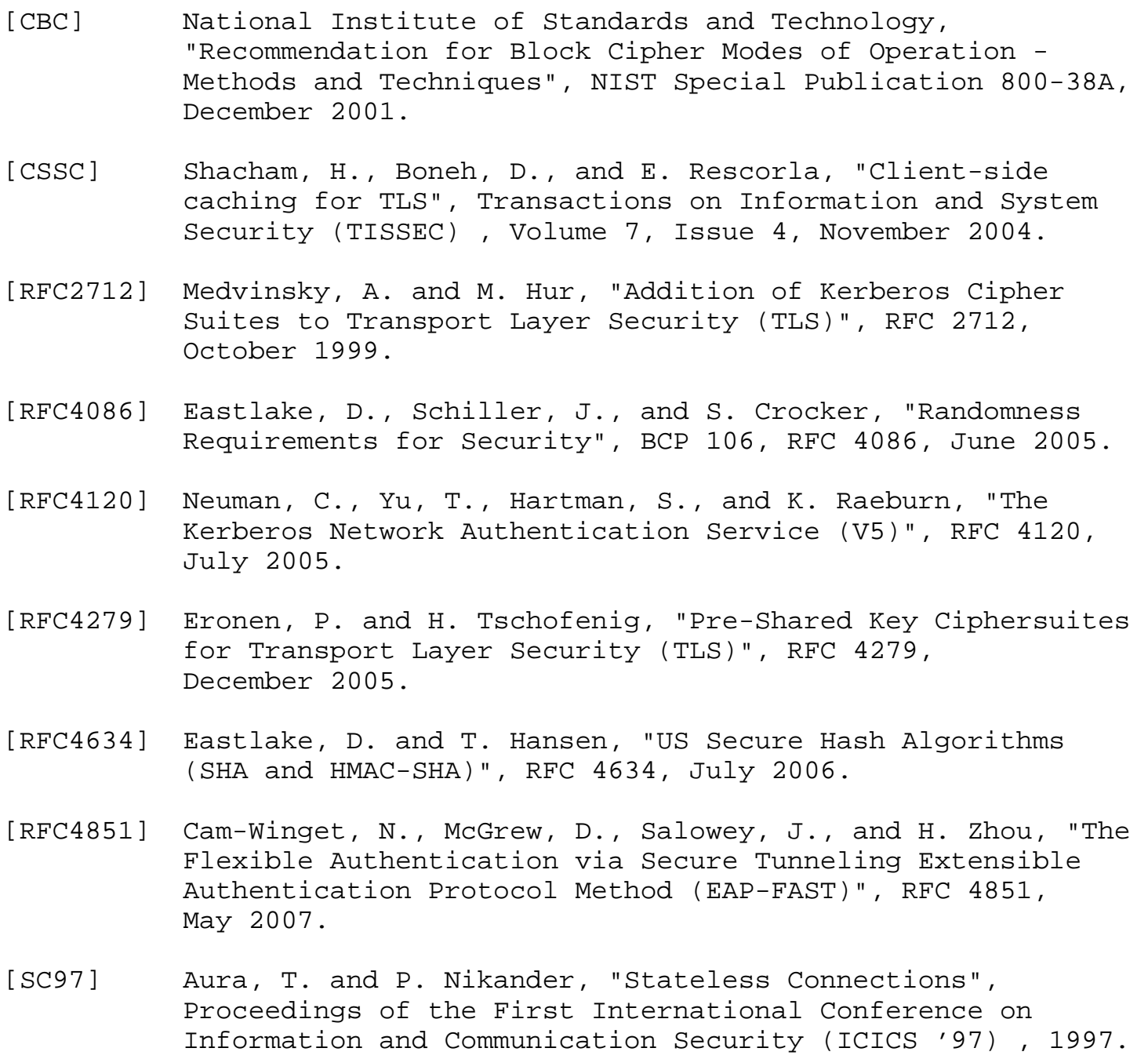


Appendix A. Discussion of Changes to RFC 4507

RFC 4507 [RFC4507] defines a mechanism to resume a TLS session without maintaining server side state by specifying an encrypted ticket that is maintained on the client. The client presents this ticket to the server in a sessionticket hello extension. The encoding in RFC 4507 used the XDR style encoding specified in TLS [RFC 4346].

An error in the encoding caused the specification to differ from deployed implementations. At the time of this writing there are no known implementations that follow the encoding specified in RFC 4507. This update to RFC 4507 aligns the document with these currently deployed implementations.

Erroneous encoding in RFC 4507 resulted in two length fields; one for the extension contents and one for the ticket itself. Hence, for a ticket that is 256 bytes long and begins with the hex value FF FF, the encoding of the extension would be as follows according to RFC 4507 :

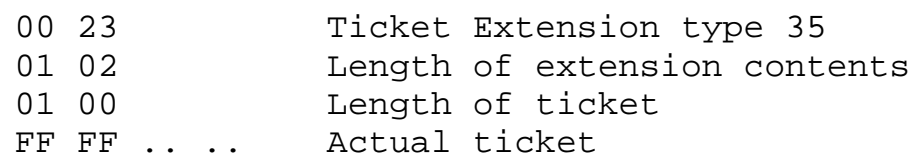

The update proposed in this document reflects what implementations actually encode, namely it removes the redundant length field. So, for a ticket that is 256 bytes long and begins with the hex value FF $\mathrm{FF}$, the encoding of the extension would be as follows according to this update:

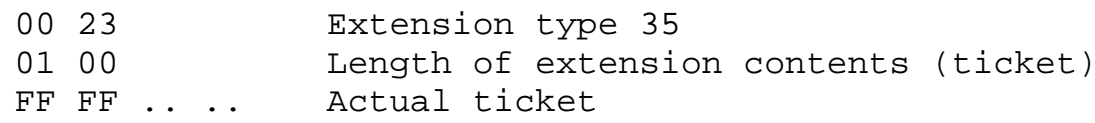

A server implemented according to $\mathrm{RFC} 4507$ receiving a ticket extension from a client conforming to this document would interpret the first two bytes of the ticket as the length of this ticket. This will result in either an inconsistent length field or in the processing of a ticket missing the first two bytes. In the first case, the server should reject the request based on a malformed length. In the second case, the server should reject the ticket based on a malformed ticket, incorrect key version, or failed decryption. A server implementation based on this update receiving an RFC 4507 extension would interpret the first length field as the 
length of the ticket and include the second two length bytes as the first bytes in the ticket, resulting in the ticket being rejected based on a malformed ticket, incorrect key version, or failed decryption.

Note that the encoding of an empty Sessionticket extension was ambiguous in RFC 4507. An RFC 4507 implementation may have encoded it as:
$00 \quad 23$
Extension type 35
$00 \quad 02$
Length of extensior
0000
Length of ticket

or it may have encoded it the same way as this update:
0023
Extension type 35
$00 \quad 00$
Length of extension contents

A server wishing to support RFC 4507 clients should respond to an empty SessionTicket extension encoded the same way as it received it.

A server implementation can construct tickets such that it can detect an RFC 4507 implementation, if one existed, by including a cookie at the beginning of the tickets that can be differentiated from a valid length. For example, if an implementation constructed tickets to start with the hex values $F F F F$, then it could determine where the ticket begins and determine the length correctly from the type of length fields present.

This document makes a few additional changes to RFC 4507 listed below.

- Clarifying that the server can allow session resumption using a ticket without issuing a new ticket in section 3.1.

- Clarifying that the lifetime is relative to when the ticket is received in section 3.3 .

- Clarifying that the NewSessionTicket handshake message is included in the hash generated for the Finished messages in Section 3.3.

- Clarifying the interaction with TLS Session ID in section 3.4.

- Recommending the use of SHA-256 for the integrity protection of the ticket in section 4.

- Clarifying that additional data can be included in the StatePlaintext structure in section 4 . 


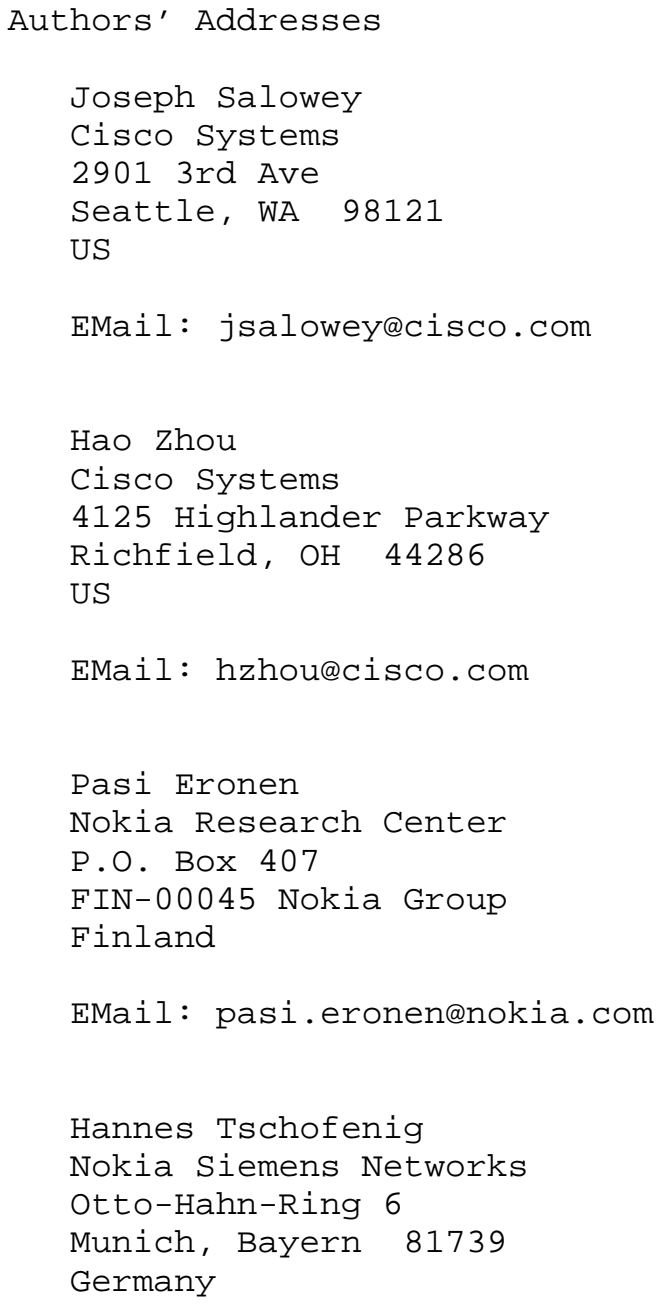

EMail: Hannes.Tschofenig@nsn.com 
Full Copyright statement

Copyright (C) The IETF Trust (2008).

This document is subject to the rights, licenses and restrictions contained in BCP 78, and except as set forth therein, the authors retain all their rights.

This document and the information contained herein are provided on an "AS IS" basis and THE CONTRIBUTOR, THE ORGANIZATION HE/SHE REPRESENTS OR IS SPONSORED BY (IF ANY), THE INTERNET SOCIETY, THE IETF TRUST AND THE INTERNET ENGINEERING TASK FORCE DISCLAIM ALL WARRANTIES, EXPRESS OR IMPLIED, INCLUDING BUT NOT LIMITED TO ANY WARRANTY THAT THE USE OF THE INFORMATION HEREIN WILL NOT INFRINGE ANY RIGHTS OR ANY IMPLIED WARRANTIES OF MERCHANTABILITY OR FITNESS FOR A PARTICULAR PURPOSE.

Intellectual Property

The IETF takes no position regarding the validity or scope of any Intellectual Property Rights or other rights that might be claimed to pertain to the implementation or use of the technology described in this document or the extent to which any license under such rights might or might not be available; nor does it represent that it has made any independent effort to identify any such rights. Information on the procedures with respect to rights in RFC documents can be found in BCP 78 and BCP 79 .

Copies of IPR disclosures made to the IETF Secretariat and any assurances of licenses to be made available, or the result of an attempt made to obtain a general license or permission for the use of such proprietary rights by implementers or users of this specification can be obtained from the IETF on-line IPR repository at http://www.ietf.org/ipr.

The IETF invites any interested party to bring to its attention any copyrights, patents or patent applications, or other proprietary rights that may cover technology that may be required to implement this standard. Please address the information to the IETF at ietf-ipreietf.org. 